Marquette University

e-Publications@Marquette

Physician Assistant Studies Faculty Research and

Publications

Physician Assistant Studies, Department

2-18-2011

Second Degree Atrioventricular Block

James F. Ginter

Aurora Cardiovascular Services

Patrick Loftis

Marquette University, patrick.loftis@marquette.edu

Published version. em > Journal of the American Academy of Physician Assistants, Vol. 24, No. 2 (February 2011), Permalink. (C) 2011, American Academy of Physician Assistants and Haymarket Media Inc. Used with permission. 


\section{Second-degree atrioventricular block}

James F. Ginter, MPAS, PA-C, Patrick Loftis, PA-C, MPAS, RN

February 182011

Unlike first-degree atrioventricular (AV) block in which the atrial impulse is delayed in reaching the ventricles, some atrial impulses in second-degree AV block fail to reach the ventricles. There are three types of seconddegree atrioventricular block: Mobitz type I, Mobitz type II, and advanced. In Mobitz types I and II, two or more consecutive P waves are conducted to the ventricle, and only one P wave is blocked. In advanced second-degree AV block, two or more consecutive P waves are blocked. On the ECG, Mobitz type I, also called Wenckebach block, is characterized by progressive PR prolongation that culminates in a nonconducted P wave. In Mobitz type II, the PR interval that precedes the blocked P wave remains constant. In both types, the atrioventricular block is intermittent and generally repetitive. Types I and II second-degree AV block differ in the location of the block and in the likelihood for progression to complete heart block.

Symptoms Second-degree AV blocks typically do not produce symptoms. If the sinus rate is low and the ratio is low (2:1, 3:2), the patient may experience a reduction in cardiac output and symptoms of hypoperfusion.

Etiology Second-degree AV blocks are usually caused by a structural disease, such as sclerosis or calcification of the conduction system as a result of aging. The block may result from ischemia, infarction, sarcoid, or amyloid, or it may occur following cardiac surgery or ablation. Second-degree atrioventricular blocks can occur in patients who have no health issues; athletes, whose hearts are exposed to more-than-usual stresses; patients taking AV nodal-blocking medications, such as digoxin, verapamil, diltiazem, and beta blockers; and patients with intrinsic atrioventricular nodal disease, acute inferior infarction, or ischemia.

\section{Mobitz type I atrioventricular block}

Wenckebach block usually involves the AV node and is considered benign. The ECG findings of a patient with type I Mobitz block (Figure 1) will show the following:

- Conduction of two or more consecutive P waves

- Blockage of a single P wave

- Gradual prolongation of the PR interval 
- Consistent shortening of the PR interval after the blocked P wave

• $\quad$ Variable and typically decreasing R-R intervals.

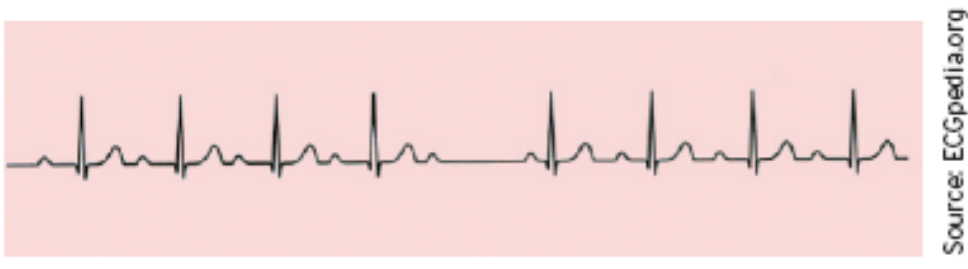

FIGURE 1. ECG of a patient with Mobitz type I atrioventricular block showing gradual prolongation of the PR interval, consistent shortening of the PR intervals after the blocked $P$ wave, and variable and decreasing $R-R$ intervals.

Conduction ratios report the number of $\mathrm{P}$ waves conducted relative to the total number of QRS complexes conducted. In Mobitz type I atrioventricular block, the conduction ratios are usually 3:2, 4:3, or 5:4. When conduction ratios are higher than 7:6, their consequences become unpredictable.

Treatment When treating a patient with AV nodal conduction delay, look first for reversible causes, such as medications, increased vagal tone, and ischemia, and correct them. If no reversible causes are found, avoid medications that slow nodal function, if possible. Patients with symptoms require a pacemaker.

\section{Mobitz type II atrioventricular block}

The site of a type II atrioventricular block is almost always below the AV node. Patients with type II atrioventricular block can develop complete heart block suddenly and without warning.

The ECG tracing of a patient with Mobitz type II AV block (Figure 2) will show the following:

- Conduction of two or more consecutive P waves

- Blockage of a single P wave

- Uniform PR intervals

- $\quad$ QRS complexes that are usually wide

- Uniform R-R intervals.

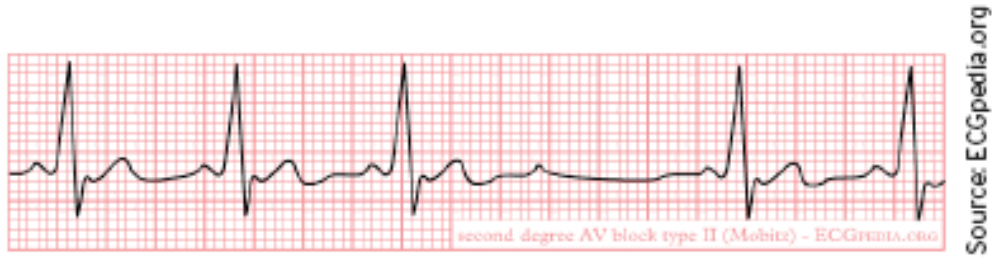

FIGURE 2. ECG of a patient with Mobitz type II atrioventricular block showing uniform PR intervals, wide QRS complexes, and uniform R-R intervals.

Treatment Mobitz type II atrioventricular blocks occur infranodally. Therefore, even asymptomatic patients require pacemaker insertion.

\section{Advanced second-degree atrioventricular block}


When the conduction ratio of a second-degree atrioventricular block is 2:1, 3:1, or higher, the block cannot be classified as type I or type II because only a single P wave is conducted. Moreover, a conduction ratio of 2:1 makes distinguishing between type I and type II difficult. These blocks and those with a conduction ratio of 3:1 or higher are categorized as advanced. On an ECG, the QRS complex can be narrow or wide. A narrow QRS complex indicates that the block is in the AV node, while a wide QRS complex indicates a block below the node.

Treatment Pacemaker insertion is indicated for symptomatic patients with advanced second-degree atrioventricular block. Many asymptomatic patients also will require pacemaker placement.

\section{ECG challenge}

Figure 3 shows the ECG of an 18-year-old cross-country runner who underwent a physical examination prior to entering the military. (To see a larger version of any figure in this article, click here.)

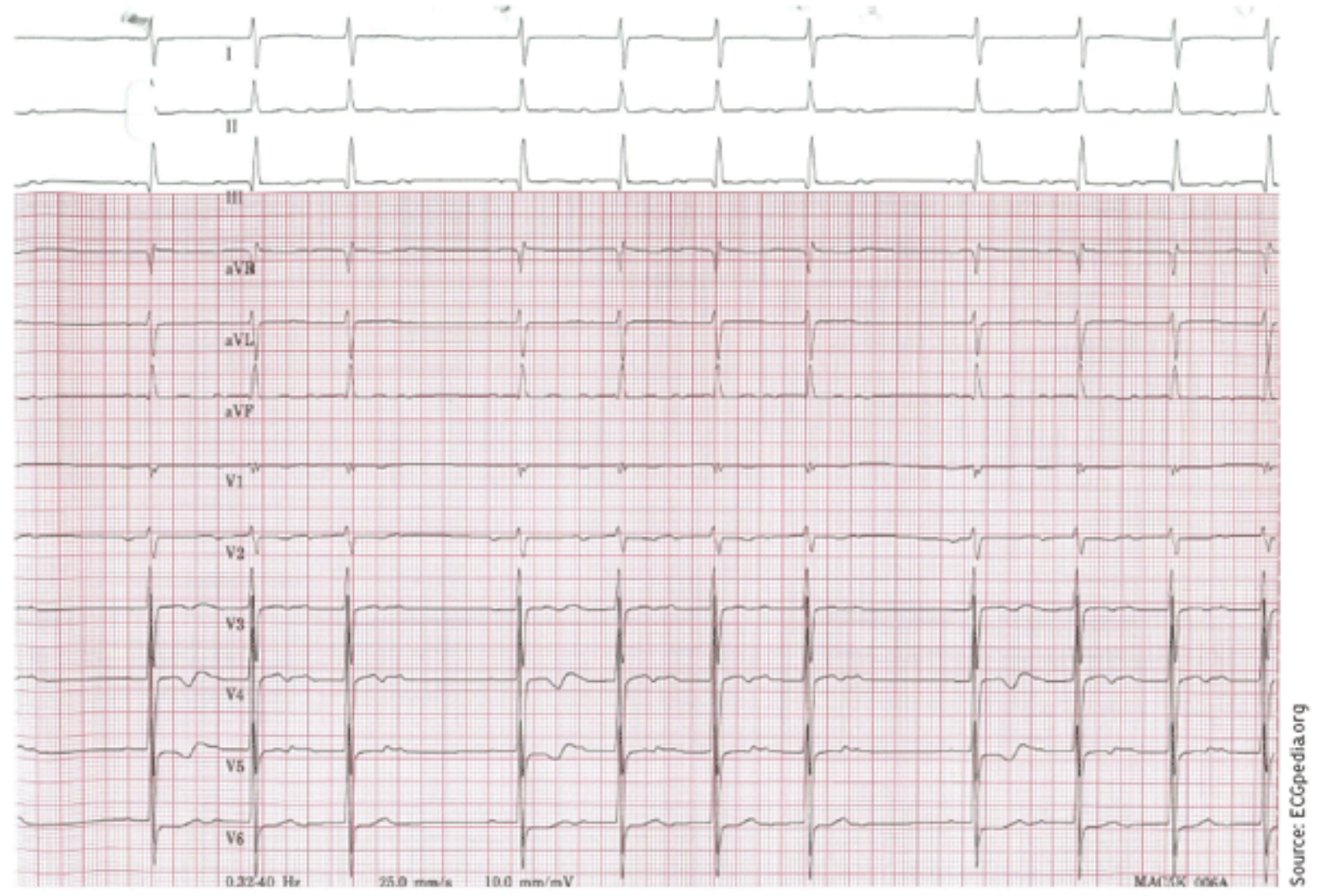

FIGURE 3. ECG of an athlete applying to the military

Using the stepwise approach to ECG evaluation, note the following:

1. Is the rhythm regular? No. There is grouped beating.

2. Estimate the rate. Since the heartbeat is irregular, use the 6-second rule of counting for a more accurate estimation of rate. Six QRS complexes occur in 6 seconds (30 large boxes), for an estimated rate of $6 \times 10$, or 60 beats per minute.

3. Is there a $\mathbf{P}$ wave before every QRS? No. Some $\mathrm{P}$ waves do not have a QRS after them.

4. The PR interval varies from 200 milliseconds to 240 milliseconds to 360 milliseconds to a nonconducted $\mathrm{P}$ wave. The cycle then repeats itself.

5. Do the QRS complexes span fewer than three small boxes? Yes. The QRS complexes are normal. 
6. ST segments are normal.

7. T waves are normal.

8. There are no $\mathbf{U}$ waves.

This ECG tracing indicates a Mobitz type I second-degree atrioventricular block. JAAPA

Jim Ginter practices at Aurora Cardiovascular Services in Milwaukee, Wisconsin. Patrick Loftis practices emergency medicine and is a clinical assistant professor in the Department of Physician Assistant Studies, Marquette University, Milwaukee, Wisconsin. The authors have no relationships to disclose relating to the contents of this article. 